\title{
Tecnología hidráulica y diseño del sistema de regadío de la cuenca del Riu Amadorio (1600-1960)
}

\author{
Pablo Giménez-Font \\ Instituto Interuniversitario de Geografia, Universitat d'Alacant \\ pablo.gimenez@ua.es
}

\begin{abstract}
Resumen. La presente comunicación quiere destacar el papel que la tecnología hidráulica (especialmente en su vertiente innovadora desde los inicios del siglo XVII) tuvo en la formación del sistema regadío de la cuenca del Riu Amadorio, que recoge las aguas de la vertiente meridional de la Serra d'Aitana (Alicante). El diseño se realizó a partir de la creación de una nueva población medieval en la costa (Vilajoiosa) y las principales innovaciones tecnológicas derivaron de la construcción de la presa de Relleu (en un interesante capítulo en el proceso de difusión de las presas en arco) y los intentos de captación del acuífero a través de la Font de l'Arc que alimentaba al Riu de Sella, principal afluente del Amadorio.
\end{abstract}

Palabras clave: Regadío, Pantano de Relleu, acuífero cárstico, ríos Amadorio y de Sella (Alicante, España)

\begin{abstract}
This paper wants to emphasize the role that hydraulic technology had (especially in its innovative side from the beginning of 17 th century) in the design of the irrigation system in Amadorio river basin, which collects the water of the southern slope of Aitana mountain range (Alicante). The design was made from the creation of a new medieval settlement on the coast (Vilajoiosa) and the main technological innovations derived, on the one hand, from the construction of the Relleu dam (in an interesting chapter in the spreading process of arc dams) and, on the other hand, from the attempts of capture the aquifer through the Font de l'Arc, which provided river Sella, the principal tributary of Amadorio.
\end{abstract}

Keywords: Irrigation, Relleu dam, karst aquifer groundwater, Amadorio and Sella river's (Alicante, Spain)

\section{Introducción}

El Riu Amadorio y su principal afluente, el Riu de Sella, se nutren de las aguas de la vertiente meridional del macizo calcáreo dominado por la Sierra de Aitana (1.558 msnm.), razón por la cual los aportes de caudales subterráneos son muy importantes a pesar de que se conceptúe como un río-rambla. Con una cuenca de unos $205 \mathrm{~km}^{2}$, presenta un módulo absoluto reducido $\left(0,2 \mathrm{~m}^{3} / \mathrm{s}\right)$ y un intenso aprovechamiento histórico que lo convierte en un interesante caso de estudio, a pesar de no haber recibido la atención merecida de investigaciones anteriores sobre el regadío valenciano. La cabecera de la cuenca se caracteriza por la presencia de un conjunto de micro y mesosistemas de regadíos en pequeñas terrazas encajadas que, como mínimo, tienen un origen andalusí. Sin embargo, el principal aprovechamiento de los caudales se haya centralizado por el macrosistema de regadío que forma la huerta de Vilajoiosa, desarrollado desde la edad Media en el glacis-cono de su tramo final antes de su desembocadura en el Mediterráneo. Su principal particularidad reside en que se trata, superada la barrera pluviométrica que determina el Cap de la Nao, del sistema de riego más septentrional -a excepción del Rec de l'Alfàs, más reciente- donde aparece la 
disociación entre la propiedad del agua y la tierra, tan característica de los regadíos del sureste peninsular(Gil Olcina, 1993).

A partir de estas particularidades, el objetivo de esta aportación es ofrecer, a modo de síntesis tras años de investigación de archivo y campo ${ }^{1}$, una reflexión sobre la importancia de la tecnología hidráulica en la captación y almacenamiento de los exiguos caudales de la cuenca y, por tanto, en el proceso de diseño y construcción de este sistema de regadío.

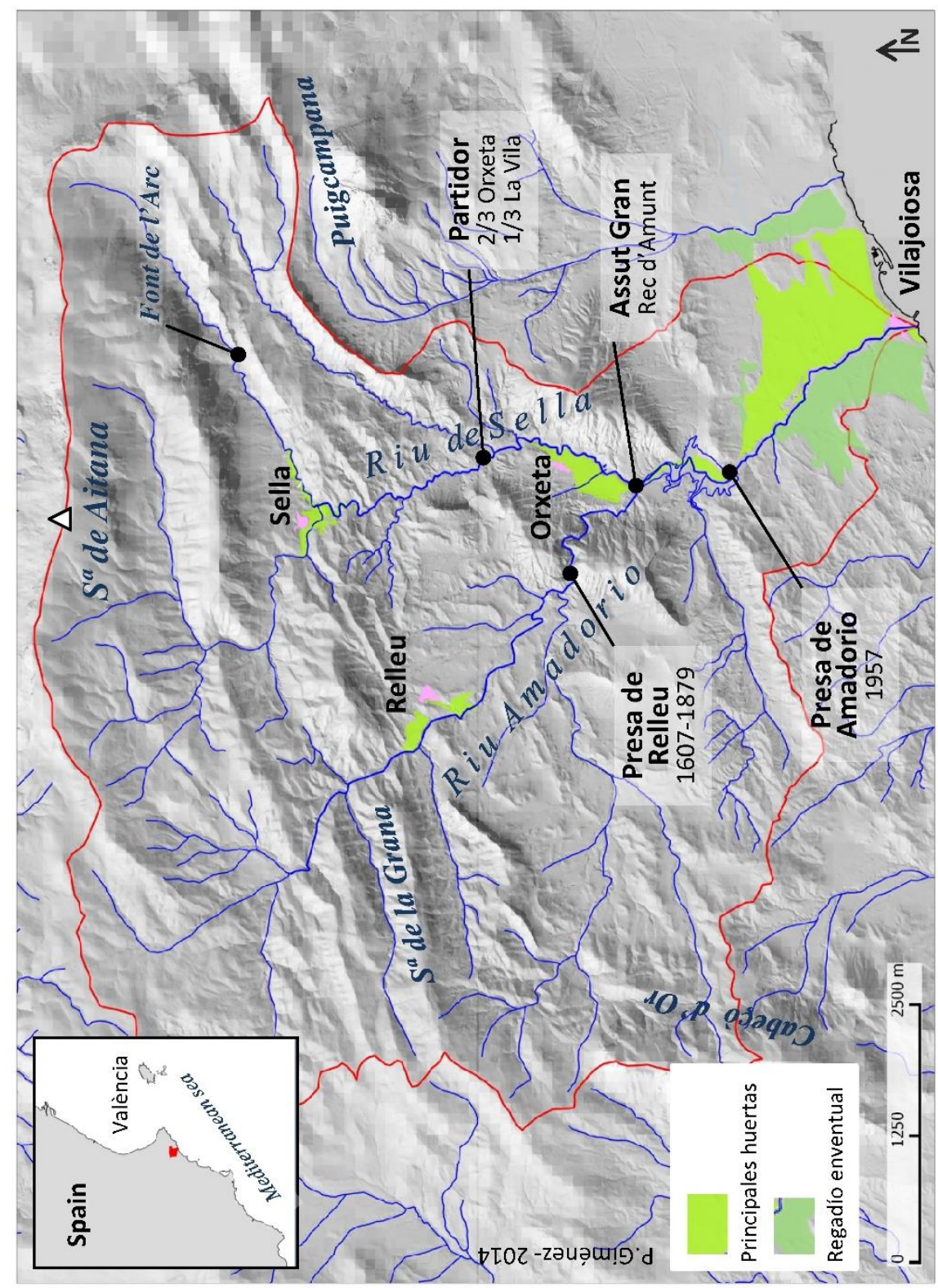

Fig. 1. Localización de la cuenca del Amadorio y principales obras citadas en el texto.

\footnotetext{
${ }^{1}$ Los archivos consultados han sido Arxiu de la Corona d'Aragó (ACA), Archivo Histórico Nacional (AHN), Arxiu del Regne de València, Archivo Histórico Provincial de Alicante y Arxiu Municipal de La Vila Joiosa.
} 


\section{La nueva población medieval de Vilajoiosa y el diseño del sistema de regadío}

Tal y como ha apuntado Thomas F. Glick (2007: 22) las transformaciones feudales del paisaje andalusí en España se realizaron a lo largo de una extensa cronología que en algunos sectores alcanzó los inicios del siglo XVII, cuando la expulsión de los moriscos repercutió en una reorganización territorial de aquellos espacios generalmente marginales donde habían sobrevivido estas comunidades. En la línea de este planteamiento, en la cuenca del Amadorio los principales cambios se produjeron a partir de 1300, cuando se funda la nueva población cristiana de Vilajoiosa en su desembocadura, y no se desarrollan plenamente hasta que no se consolidan -ya en la segunda mitad del siglo XVIII- las comunidades rurales postmoriscas del interior, sujetas a la jurisdicción señorial. En ese intervalo de tiempo, convertida en villa real y con una creciente actividad portuaria, Vilajoiosa dispuso de sustanciosas ventajas para atraer y asentar población, adquiriendo plenamente su condición de capital comarcal gracias a los privilegios que le fueron concedidos en su fundación. Dichos privilegios le permitían controlar recursos naturales de la vertiente sur de la sierra de Aitana como los pastos, las leñas, la extracción de madera y, especialmente, el agua (Galiana, 2011). La población costera dispuso de pastos de verano en las sierras de Orxeta y Aitana, aprovechó la madera de los bosques interiores para sus prestigiosos calafates y centralizó el aprovechamiento de los recursos hídricos de la cuenca conforme la coyuntura socioeconómica y técnica le fue favorable.

\subsection{Tentativa de cronología}

La tentativa de establecer una cronología parte de la necesidad de sistematizar la formación de un sistema de regadío realizado desde el litoral, concretamente a partir de los inicios del siglo XVII.

Esta fecha señala un cambio determinante, puesto que, como se ha dicho, la expulsión de los moriscos que habitaban casi exclusivamente el interior montañoso permitió consolidar la organización territorial proyectada con la conquista cristiana. En paralelo, Vilajoiosa conoció la disminución de la presión ejercida por los ataques piratas, constantes en el s. XVI, e intensificó el comercio marítimo, aumentando el número de habitantes y la agricultura.

Partiendo de esta premisa y a partir de una cronología aproximativa, se han establecido cuatro grandes periodos o fases de construcción del sistema de regadío que pretenden facilitar la perspectiva general de su evolución:

- Construcción de la presa de Relleu y consolidación (1600 - 1730): la primera proyección de la obra, a cargo de Cristobal Antonelli, data de 1607, aunque el privilegio real no se otorga hasta 1653 y la presa no parece funcionar hasta, al menos, 1689. Las estimaciones calculaban duplicar la superficie regada (de 115 a $230 \mathrm{ha}$ ), aunque la presa no se culminó completamente y los cambios en los cultivos no fueron significativos. En este periodo se inician también las primeras obras de captación de la Font de l'Arc. Por el momento, desconocemos a partir de qué momento la propiedad del agua se separó de la de la tierra.

- Intensificación y búsqueda de caudales alternativos (1730-1860): durante este período se produjo el principal incremento de las superficies roturadas en el conjunto de la cuenca, lo que repercutió en una intensificación de los usos del agua mediante un crecimiento exponencial de la pequeña hidráulica. En 1772, se inician las obras para aumentar la altura de la pared del pantano y se incrementa la superficie regada hasta las aproximadamente 300 ha. A principios del siglo XIX un particular 
primero y la unión de más de 150 regantes después, adquieren la Font de l'Arc e incrementan las obras de captación de su caudal. A pesar de no obtener el éxito esperado, se suceden los proyectos de ampliación del regadío en Vilajoiosa, especialmente en la margen derecha del río, todavía de secano. Se incrementa la conflictividad hidráulica en torno al partidor de aguas de Orxeta.

- Estancamiento y proyectos supramunicipales (1860-1960): los problemas de colmatación de la presa de Relleu y la infructuosa búsqueda de caudales en la Font de l'Arc, en un contexto de crecimiento agrario y desarrollo de nuevos cultivos comerciales como la vid o los cítricos, obligaron a la búsqueda de soluciones dentro de los programas de planificación hidrológica estatal. Es el caso del Canal del Algar de Francisco Morell (de 1866, incluido en el Plan Gasset de 1902) o los intentos de conectar la Font de l'Arc (con estación de bombeo desde 1937) con el pantano de Relleu, tal y como se propuso en el Plan Nacional de Obras Hidráulicas de Lorenzo Pardo (Mateu, 2010). Finalmente, se optó por la construcción de un nuevo embalse en la confluencia de Amadorio y Sella, que se inauguró en 1956.

- Nuevos regadíos y nuevos usos del agua (1960-2000): la construcción del pantano de Amadorio supuso la adscripción obligatoria de la propiedad del agua a la de la tierra y determinó profundos cambios en la extensión, la estructura del parcelario y los cultivos de la huerta de Vilajoiosa. A pesar del significativo incremento de la superficie regada hasta superar 1.240 ha en 1970 (Quereda, 1978), el crecimiento urbano-turístico y la sequía obligaron a crear el Consorcio de Aguas de la Marina Baixa, que trata de complementar los usos agrarios con los urbanos mediante la explotación conjunta de acuíferos y el intercambio de aguas limpias y residuales depuradas. En paralelo a la consolidación de esta mancomunidad, se ha incrementado el abandono de superficies agrícolas, la sustitución por coberturas urbanas y la inutilización parcial de las infraestructuras de riego tradicionales.

\section{Tecnología hidráulica}

Presentadas los principales aspectos territoriales -que no institucionales, puesto que conscientemente hemos evitado hablar de las ordenanzas de riego por razones de espacio- el proceso de crecimiento del sistema de regadío de la cuenca del Amadorio se sustenta en gran parte sobre dos desafíos tecnológicos principales: la construcción de la presa de Relleu y la explotación del acuífero meridional de Aitana a través de la Font de l'Arc.

\subsection{La presa de Relleu}

Aunque su excepcionalidad técnica ha quedado refrendada por numerosos especialistas a lo largo del siglo XX, el pantano de Relleu no ha disfrutado del reconocimiento que merece. Considerada por Schnitter (2000: 143) como la obra que marcó el final de las presas modernas en el sureste de España, el reciente descubrimiento de la presencia de Cristobal Antonelli en su proyección y su correcta datación -su origen se remonta a 1607- le aportan un papel, si cabe, más destacado en la historia de la técnica hidráulica mundial (Giménez-Font, 2014). Con todo, también cabe señalar que la importante historiografía hidráulica del sureste peninsular tampoco ha atendido suficientemente el contexto de innovación tecnológica localizada en este territorio desde finales del siglo XV hasta la primera mitad del XVII. Por esta razón, cabría preguntarse, principalmente, sobre el proceso de difusión del 
conocimiento y la técnica que permitieron levantar un muro tan estable e innovador, que supuso un avance importante en la transición de las presas escalonadas a las de planta curva ¿fueron aplicaciones empíricas o existía una doctrina teórica previa?

Aunque todavía no estamos en disposición de responder a esta pregunta, se continúa avanzando en el estudio de las variables que, según Glick, intervienen en la difusión de las presas en arco. No cabe duda de la importancia de la vertiente endógena de este proceso, con la implicación de técnicos locales como los maestros Pedro de Aguirre, Joanes del Temple (presas de Almansa y Elche), Pere Izquierdo (proyecto inicial de Tibi), la desconocida relación entre los dos últimos, o los muchos otros técnicos valencianos de formación poco conocida implicados en estas obras. Pero, igualmente, la presencia de reconocidos técnicos europeos al servicio del imperio español permitió el movimiento de conocimiento y el trasvase de técnicas desde el campo militar -principalmente la construcción de fortalezas- a la ingeniería hidráulica. De entre todos los técnicos reconocidos, los italianos presentan una mayor versatilidad en este sentido: Juanelo Turriano, Jorge Palearo Il Fratino, Francesco Sitoni, Vespasiano Gonzaga y, especialmente, la saga de los Antonelli, una familia procedente de Gatteo que contó con siete ingenieros al servicio a la monarquía hispana durante casi un siglo. Cristobal Garavelli Antonelli (1550-1608) acompañó a su tío Juan Bautista y a Jorge Palearo durante los inicios de la construcción de la presa de Tibi, pero pronto se encargó de la dirección de las obras, aplicando mejoras sobre el proyecto inicial y dejando un extenso catálogo de planos al respecto (González Gascón, 1999). Las semejanzas entre esta presa y la de Relleu no quedan únicamente en el plano operativo iniciativa local con apoyo de la Corona, término municipal diferente al promotor, riego de secanos mejorados- sino también en el plano técnico: Tibi resultó revolucionario por su planta curva y por su altura, nunca alcanzada antes; Relleu, el último proyecto antes de fallecer Antonelli, resultó el más esbelto del mundo durante dos siglos, al combinar altura y espesor uniforme empleando también el efecto arco en transición hacia la bóveda. Ambas obras, levantadas cual murallas sobre cerradas calizas, parecen representar un proceso de innovación tecnológica que todavía espera un estudio comparativo detallado.

Con todo, la edificación de Relleu no se concretó de forma tan rápida y decisiva como en el caso de Tibi, puesto que el proyecto original no debió iniciarse hasta después de la firma del privilegio real de 1653 y en distintas fases: 22,5 $\mathrm{m}$ a finales del s. XVII; 24,5 $\mathrm{m}$ a finales del XVIII (¿altura del proyecto original?) y los actuales $32 \mathrm{~m}$ de finales del XIX.

Tampoco se pudo solucionar correctamente el desafío técnico que supuso la evacuación de lodos en estos embalses, que progresivamente fueron mermando su capacidad, a pesar de los proyectos de recrecimiento planteados hasta 1911, que preveían incrementar la altura hasta los $44 \mathrm{~m}$. 



Fig. 2. Las presas de Relleu (s. XVII) y Amadorio (1956), representan cuatro siglos de gestión hidráulica dirigida desde Vilajoiosa. 


\subsection{La captación de la Font de l'Arc}

Capítulo complementario a la construcción de la pared en Relleu fueron los intentos de captación del principal acuífero de la cuenca, que alimentaba el Riu de Sella y cuya surgencia principal se realizaba a través de la Font de l'Arc - uno de los pocos topónimos de origen latino que sobrevivieron al dominio islámico-, localizada en los límites de los términos municipales de Sella y Benimantell.

El aprovechamiento de los recursos hídricos provenientes de la descarga de este acuífero merece una mención directa en la carta puebla medieval de Vilajoiosa (1300), lo cual resulta indicativo del interés vilero por el control de la fuente. En la documentación estudiada, creciente en número a partir del siglo XVII, las referencias a los caudales previsibles del acuífero son constantes y anuncian un problema de percepción que deriva del propio comportamiento fugaz y espasmódico de una surgencia cárstica de tipo trop plein (Marco Molina, 2001 y Hermosilla, 2006), capaz de arrojar más de 2.300 l/ s². La creencia de la existencia de enormes caudales ocultos en el corazón de Aitana animó ambiciosas empresas que tienen su origen, al menos, en la década de 1690, cuando diversos canteros de la población litoral excavaron una mina en busca del nacimiento de las aguas 3 . Aunque hay constancia de la presencia de un experto hidráulico italiano en Relleu durante la segunda mitad del XVIII ${ }^{4}$, las referencias hasta el momento indican la participación de expertos locales en la construcción de la mina. En 1804 la fuente fue adquirida a la baronía de Benimantell por un particular de la Vilajoiosa, que proyectó la puesta en regadío eventual de la margen derecha del Amadorio (unas 670 ha), gracias a un azud construido en 1796. Arruinado en la búsqueda de agua, en 1822 la fuente y sus terrenos adyacentes fueron adquiridos por una sociedad constituida por más de 150 agricultores, que prosiguió su búsqueda sin éxito -realizando la galería inferior en 1850- hasta la segunda mitad del siglo XX, ya como comunidad de regantes (Giménez-Font, 2008). Con un conjunto de galerías que superan los $150 \mathrm{~m}$ de longitud, conectadas entre sí por un pozo de $22 \mathrm{~m}$, en 1937 después de recibir la visita del ministro de obras públicas Indalecio Prieto- se consiguió instalar la primera bomba de extracción a gasóleo y posteriormente se electrificó, pero los resultados nunca fueron los esperados. El desarrollo de los estudios hidrogeológicos y el establecimiento de sondeos de ensayo durante la década de 1990 han permitido conocer de una forma más precisa los límites y niveles piezométricos del acuífero (Rodríguez Estrella, 2002), descartando la capacidad de almacenamiento que se le presuponía desde época medieval.

\footnotetext{
${ }^{2}$ Se trata del caudal máximo medido (año 1991), en una corta serie inferior a los 40 años (IGME, 2008)

${ }^{3}$ ACA. Consejo de Aragón, Legajo 673, nº 21 y Legajo 860, no 26

${ }^{4}$ Antonio Ferrari Caracciolo. AHN. Consejos Legajo 37.165 (1789)
} 

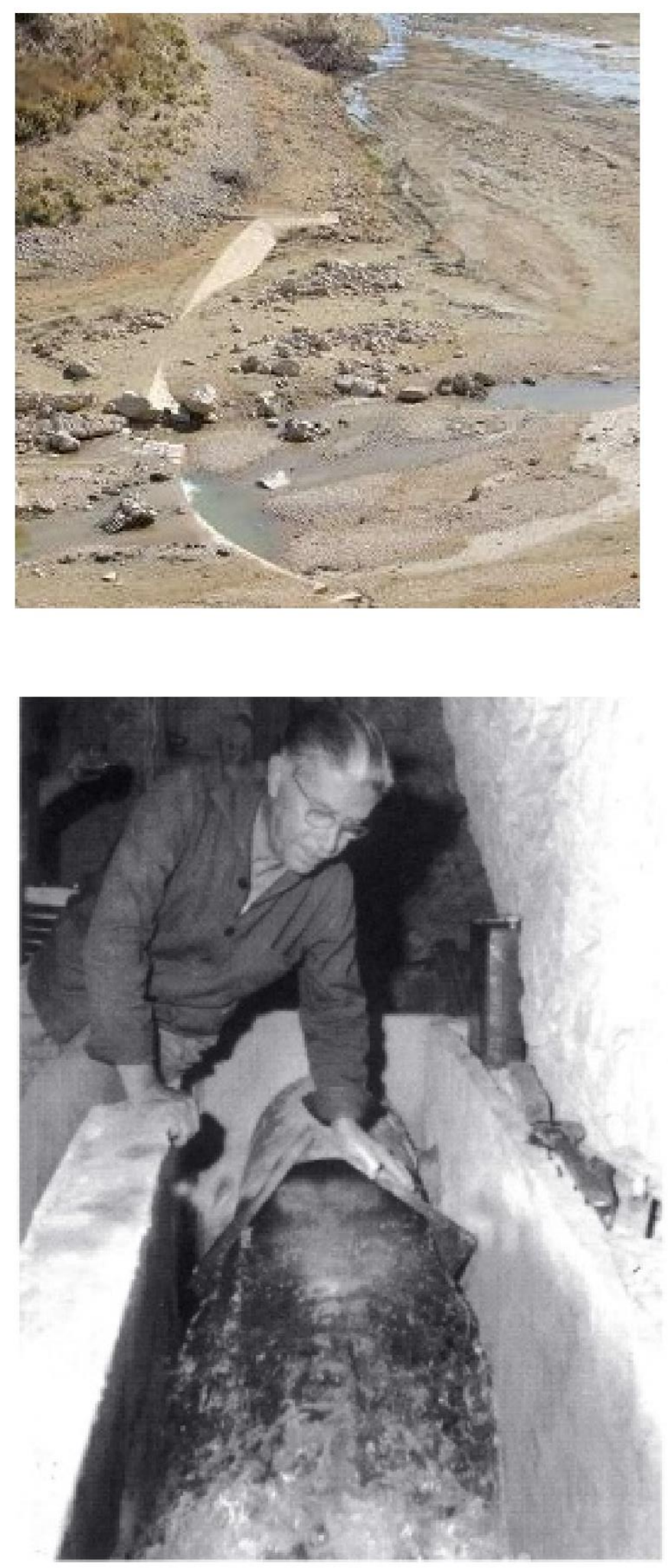

Figs. 3 y 4. Bombeo electrificado en la galería inferior de la Font de l'Arc (en torno a la década de 1960), cuya construcción se inició en la década de 1840, al igual que la ampliación de l'Assut Gran (arriba), hoy bajo las aguas del pantano de Amadorio. 


\subsection{Hacia el aprovechamiento integral de las aguas: ss. XIX $\mathrm{Y} X X$}

Las expectativas creadas en torno a la presa de Relleu y la explotación del acuífero de Aitana, fallidas a lo largo de varios siglos por las dificultades técnicas que entrañaba la retención de tarquines, las filtraciones y los problemas de captación de las aguas subterráneas, evolucionaron hacia medidas convergentes de aprovechamiento integral de la cuenca. La posibilidad de hacer llegar las aguas de la fuente, una vez instalada la bomba de extracción, al principal reservorio de la huerta vilera tomó forma de la mano de la propuesta de José Marqués Lis (1934), Ingeniero Jefe de Aguas de la Delegación de los Servicios Hidráulicos del Júcar, que planteaba el Canal de Sella para lograr este fin, a partir de una estación de elevación. Marqués confería a dicha obra un carácter preferente dentro del conjunto de propuestas complementarias al Plan Nacional de Obras Hidráulicas de Lorenzo Pardo, aunque en este ya se recogía la posibilidad -fundamentada en la década anterior- de construir un nuevo pantano aguas abajo que aprovechara de forma más directa las aguas de Aitana (Mateu, 2010).

Recordemos que las aguas del pantano de Relleu y las del l'Arc confluían en un nudo hidrográfico formado por la conexión de los ríos Amadorio y de Sella, hoy en la cola del embalse de Amadorio. Allí se localizaba el Assut Gran, un doble azud ampliado en torno a 1848 donde nacía el denominado Rec d'Amunt de Vilajoiosa y que en la actualidad se encuentra sepultado por los sedimentos del nuevo pantano. En este punto la Confederación Hidrográfica del Júcar instaló, entre 1912 y 1915, dos estaciones de aforo que estuvieron activas hasta 1945 (Mateu, et al. 2012), poco antes de comenzar las obras del nuevo pantano. Inaugurado en 1956 con una capacidad inicial prevista de $16 \mathrm{hm}^{3}$, el embalse desvirtuó radicalmente el sentido y funcionalidad del sistema de gestión de las aguas, diversificado más tarde con la llegada de caudales no convencionales y trasvases intercuenca en un contexto de creciente desarrollo urbano-turístico.

\section{Perspectivas}

La tecnología hidráulica, en relación con el almacenamiento y la captación de caudales, ha querido dirigir el desarrollo de este planteamiento como una pieza básica de la construcción del sistema de regadío analizado. Pero, obviamente, se trata de un elemento más del complejo conjunto de interrelaciones que lo explica (Pérez-Picazo, 2000). Los factores de orden político y jurisdiccional, que hunden sus raíces en la conquista cristina medieval y el proceso de reorganización territorial que ésta conllevó, permiten entender cómo el diseño del sistema se dirigió desde Vilajoiosa. Esta población de realengo contó con los privilegios necesarios para ello, pero en la gestión del recurso hídrico demostró también una gran cohesión social que permitió las audaces gestiones que posibilitaron la construcción de dos presas en cuatro siglos. La captación y control del acuífero meridional de Aitana, a pesar de provenir de unas expectativas sobredimensionadas durante siglos, es otra demostración de la fuerza adquirida por una población obligada, ante las carencias hídricas, a diversificar su economía (pesca, calafates, esparto o chocolate). Los resultados nunca fueron los esperados, precisamente, por problemas de tipo técnico; pero el entarquinamiento de la presa de Relleu y el infructuoso minado de la Font de l'Arc no deben desvirtuar el valor tecnológico de estas obras, opciones impulsadas en un marco de gestión hidráulica fundamentalmente local. Como se ha dicho en repetidas ocasiones, la innovación no puede considerarse como algo eminentemente exógeno, ya que su aplicabilidad fue posible, en parte, gracias a la presencia de saberes o cualificaciones tradicionales y a determinados contextos socio-económicos en la gestión del agua. Nuevos estudios sobre cuencas fluviales como la 
presente podrían aportar datos relevantes sobre la vertiente endógena del progreso técnico, tanto desde el punto de vista de la construcción de las presas modernas como el de la captación de acuíferos, en un contexto de innovación y difusión que ocupó las tierras del SE Ibérico desde finales del siglo XVI.

\section{Referencias}

Galiana, A. (2011): La fundació de Vilajoiosa per Bernat de Sarrià, Sarrià, 6:4-38

Gil Olcina, A. (1993): La propiedad de las aguas en el sureste peninsular, Alicante, Universidad.

Giménez-Font, P. (2003): El Pantano de Relleu y el riego de la huerta de Villajoyosa (1653-1879), Investigaciones Geográficas, 30:97-118.

Giménez-Font, P. (2008): Las transformaciones del paisaje valenciano en el siglo XVIII. Una perspectiva geográfica, València, Alfons el Magnànim

Giménez-Font, P. (2014): Cristobal Antonelli y la innovación de la presa-bóveda de Relleu, en A. Rico y J. Olcina, Ed., Libro homenaje a Antonio Gil Olcina, Universidad de Alicante.

Glick, T.F. (2007): Paisajes de conquista. Cambio cultural y geográfico en la España medieval, Valencia, Universitat.

González Gascón, I. (1999): Felipe II, los ingenios y las máquinas: ingeniería y obras públicas en la época de Felipe II, Madrid, Sociedad Estatal para la Conmemoración de los Centenarios de Felipe II y Carlos V.

Hermosilla, J. Dir. (2006): Las galerías drenantes del Sureste de la Peninsula Ibérica. Uso tradicional del agua y sostenibilidad en el Mediterráneo español, Madrid, Ministerio de Medio Ambiente.

IGME (2007): Acuiferos de Sierra Aitana y su entorno (Alicante), http://www.igme.es/INTERNET/SIDIMAGENES/130000/671/130671_0000003.PD $\underline{F}$

Marco Molina, J.A. (2001): Aitana como espacio singular. Perspectiva geográfica de una montaña mediterránea, Alicante, Universidad

Mateu, J.F. (2010): La primera Confederación Hidrográfica del Júcar (1934-1942), Valencia, CHJ.

Mateu, J.F., Ruiz, J.M. y Portugués, I. (2012): Desarrollo del servicio de aforos en España. La red de estaciones de la Confederación Hidrográfica del Júcar, Valencia, CHJ.

Pérez-Picazo, M.T. (2000): Nuevas perspectivas en el estudio del agua agrícola. La subor-dinación de la tecnología a los modos de gestión, Historia Agraria, 22:37-56.

Quereda, J. (1978): Comarca de La Marina (Alicante). Estudio de Geografía Regional, Alacant, Diputación Provincial de Alicante.

Rodríguez Estrella, T. (2002): Definición geométrica del acuífero calizo eocénico, kárstico y arrecifal, de Sella (Alicante), perteneciente a la Sierra de Aitana, Geogaceta, 31:51-54

Schnitter, N.J. (2000): Historia de las presas. Las pirámides útiles, Madrid, Colegio de Ingenieros de Caminos, Canales y Puertos. 